\title{
Fully automatic spray-LBL machine with monitoring the real time growth of multilayer films using Quartz Crystal Microbalance
}

\author{
S. Shiratori, N. Fukao, K.H. Kyung \\ School of Integrated Design Engineering, Keio University, Yokohama 223-8522, Japan \\ e-mail: shiratori@appi.keio.ac.jp
}

\begin{abstract}
A fully automatic spray-LBL machine with monitoring the real time growth of multilayer films using Quartz Crystal Microbalance (QCM) techniques was newly developed. We established fully automatic spray layer-by-layer method by precisely controlling air pressure, solution flow, and spray pattern. The movement pattern towards the substrate during solution spraying allowed fabrication of a nano-scale, flat, thin film over a wide area. Optimization of spray conditions permitted fabrication of the flat film with high and low refractive indexes, and they were piled up alternatively to constitute a one-dimensional photonic crystal with near-infrared reflection characteristics. The heat shield effect of the near-infrared reflective film was also confirmed under natural sunlight. It was demonstrated that the fabrication using the automatic spray-LBL machine and real-time QCM monitoring allows the fabrication of optical quality thin films with precise thickness.
\end{abstract}

\section{INTRODUCTION}

Various thin films are used as visible optical antireflection films for glasses and windows, and also to raise the photoelectric conversion efficiency of solar batteries.[1-3] Band-pass filters that selectively transmit monochromatic light are used in cameras and photocells, along with other increasing numbers of applications.[4,5] Eco-friendly films that selectively reflect near-infrared radiation have also been developed.[6] As such films do not transmit heat in the near-infrared region, the increase in indoor temperature can be suppressed by applying these films to windows. For all the above optical applications, the precise control of film thickness and reflective index is necessary. Consequently, vapour metal deposition on substrates is typically carried out under vacuum, which generally limits the fabrication of reproducible optical thin films to industrial processes. For other applications like windows which require film formation over a wide area, the production cost under vacuum conditions can be prohibitive, since large volume vacuum containers and considerable energy to achieve vacuum are required.

The layer-by-layer (LBL) self-assembly method appears promising for overcoming the above difficulties.[1,6,7] It is an easy, aqueous-based, ecofriendly method carried out under room temperature and at $1 \mathrm{~atm}$, and has been thoroughly explored by Gero Decher et al.[8] A disadvantage was that the fabrication time was too long for many industrial applications, and consequently the idea of a spray-LBL method, where solutions were sprayed directly onto substrates, was conceived.10 Fabrication time was reported to be greatly shortened due to enhanced adsorption by the spray pressure. An automated spray LBL machine has been previously reported[9]. The original features of our apparatus are: (1) real time Quartz Crystal Microbalance (QCM) sensing and (2) separate control of spray pressure, solution flow rate and spray scanning pattern. The sprayLBL method is still an emerging field of research[10-14], and mechanisms during spraying have yet to be investigated. Uninvestigated parameters include spray pressure, adsorption time, distance between spray nozzle and substrate, diameter of nozzle, size of droplet, etc. There are reports[15-19] of QCM techniques having been used to determine film mass, but not for real-time monitoring during the spray-LBL process.

In this study, an automatic spray-LBL machine was fabricated and film reproducibility was examined. In situ monitoring was realized using QCM techniques, and the optimization of film fabrication conditions was carried out. The spray-LBL method provided sufficient film quality for their application as optical thin films. We optimized the moving pattern of the spray nozzle and other important parameters. The research of the effects of various parameters unveiled the process of the fast film fabrication by spray-LBL deposition. Also we fabricate the flat film with high/low refractive index, and they were 
piled up alternatively to constitute the 1-dimensional photonic crystal designed as Near-IR reflection character.

\section{Materials and Methods}

\subsection{Materials}

For the low reflective index layer, poly(allylamine hydrochloride) (PAH) and poly(acrylic acid) (PAA) were used as the polycations and polyanions, respectively. For the high reflective index layer, poly(diallyldimethylammonium chloride) (PDDA) was used as the polycation, and titanium (IV) bis(ammonium lactato)dihydroxide (TALH) was prepared. All materials were purchased from Sigma-Aldrich and were used without further purification.

\subsection{Fabrication of the automatic spray-LBL machine.}

There are previous reports of an automatic spray-LBL machine.[17] We have fabricated an automatic machine which could uniformly spray on wide substrates. A schematic of our machine is shown in Fig.1.

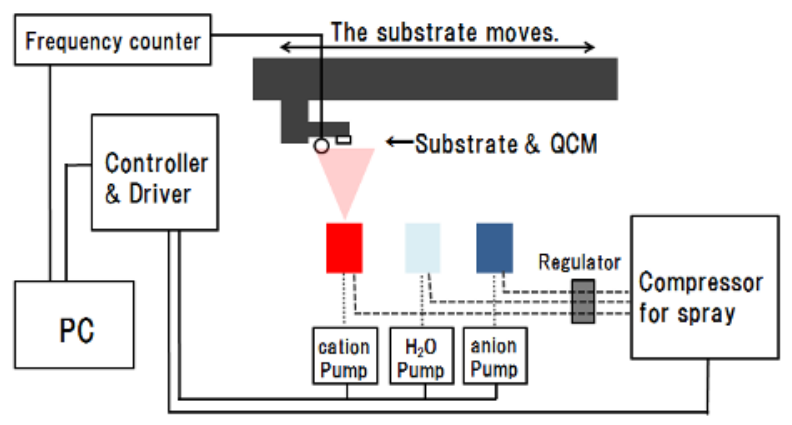

Figure 1. Automatic spray-LBL machine. Substrates and QCM were fixed on the stage, and all steps were computer controlled.

Three pumps were incorporated for the rinsing of the cationic, anionic and pure water, as it was necessary to independently change their flow rates. A regulator was used to control the spray pressure from a single compressor. Thus, spray pressure was altered by changing flow quantities. The QCM electrode was positioned near to the substrate to ensure it was also coated at the same time. QCM frequency data were counted with a frequency counter and were output to a PC for analysis. This system provided us with the real time increase in film mass. All operations such as repetition frequency and movement patterns were computer controlled. The distance from spray nozzle to substrate was fixed, and spray pressure was controlled at the proper value. A high volume compressor was utilized to maintain constant conditions and prevent pressure decrease during film fabrication.

2.3 Substrate preparation. QCM substrates were placed in an ozone atmosphere under UV irradiation for 6 hours to ensure hydrophilicity. Silicon wafers were also treated by UV irradiation for 2 hours to form a hydrophilic surface, and were used to investigate deposited film thickness and reflective index. UV irradiation removed any organic contamination present on the substrate. Glass slides were cleaned and negatively charged by using $\mathrm{KOH}$ solution.

2.4 Solution preparation. The concentrations of $\mathrm{PAH}$, PAA and PDDA solutions were all $0.01 \mathrm{~mol} / \mathrm{L}$, and that of the TALH solution was $2 \mathrm{~g} / 100 \mathrm{ml}$. Solutions were stirred overnight. Their $\mathrm{pH}$ was adjusted by adding 0.1 $\mathrm{mol} / \mathrm{L} \mathrm{NaOH}$ or $0.1 \mathrm{~mol} / \mathrm{L} \mathrm{HCl}$ (PAH:3.5, PAA:3.5, PDDA:5.5, TALH:3.5). After $\mathrm{pH}$ adjustment, solutions were used within a few hours. ${ }^{20}$

2.5 Spray conditions. Parameters were divided into fixed and variable parameters. The nozzle diameter was $1.2 \mathrm{~mm}$, and droplet size was $10-100 \mu \mathrm{m}$. Solution and water flow rates were adjusted to $4.0 \mathrm{ml} / \mathrm{min}$. The distance between substrate and spray nozzle was fixed to $15 \mathrm{~cm}$, and the sprayed area was a circle of diameter $\sim 4 \mathrm{~cm}$. Spray pressure and spray time were variable parameters. The pressure was measured nearby the spray nozzle during air flow, and controlled at $0.025,0.05$ and $0.075 \mathrm{MPa}$. Times of each step were as given in Table 1.

\begin{tabular}{|} 
Table 1. Stage times \\
\begin{tabular}{|c|c|c|}
\hline \multicolumn{2}{|c|}{ Time (s) } \\
\hline Step & Cation & Anion \\
\hline 1 Stable state & 30 & 30 \\
\hline 2 Spraying & 10 & 10 \\
\hline 3 Waiting state & 10 & 10 \\
\hline 4 Rinsing state & 10 & 10 \\
\hline 3 Drying state & 10 & 10 \\
\hline \multirow{2}{*}{ Total } & 70 & 70 \\
\cline { 2 - 3 } & \multicolumn{2}{|c|}{140} \\
\hline
\end{tabular}
\end{tabular}

For low refractive index layer (designated as $\mathbf{L}$ ), as cationic solution, poly (diallyl-dimethyl -ammonium chloride) (PDDA; Mw 200,000 300,000/10mM) was used, and as anionic solution, colloidal silica particle $\left(\mathrm{SiO}_{2}\right.$-col; $\left.\varphi 7 \mathrm{~nm} \sim / 0.1 \mathrm{wt} \%\right)$ was used. For high refractive index layer (designated as $\mathbf{H})$, cationic PDDA (50mM), and anionic titanium (IV) bis (ammonium lactato) dihydroxide (TALH; 2wt \%) was used. The cationic solution was sprayed on the negatively charged substrate (designated as G). After pure water was sprayed for removing the surplus aggregation on the surface, anionic solution was sprayed at the same conditions. The cycle was repeated n times. (we call it "n bi-layer")

\subsection{Measurements}

Film thickness was determined by ellipsometry measurements. Optical characterization of multilayer films was carried out using an ultraviolet-visible (UVvis.) spectrophotometer. Surface images were captured by field-emission scanning electron microscopy (FE-SEM;) and atomic force microscopy (AFM;). Optical simulation software was used.

\section{Results}

First of all, we checked the control accuracy of the film deposition by using basic weak polyelectrolyte PAH/PAA sysytem. [20] 


\subsection{In-situ monitoring by QCM}

Adsorption characteristics of (PAH/PAA) n films were observed by QCM (Fig.2). Frequency data were displayed as the change of frequency from a stable state (Y-axis) relative to time (X-axis). The procedure was divided into five steps: $\mathbf{1}$ stable state; $\mathbf{2}$ spraying state; $\mathbf{3}$ waiting state; $\mathbf{4}$ rinsing state; $\mathbf{5}$ drying state. These steps are also displayed in Fig.2. Total area of spray coating was $2 \mathrm{cmX} 7 \mathrm{~cm}$ on a slide glass in this experiment.

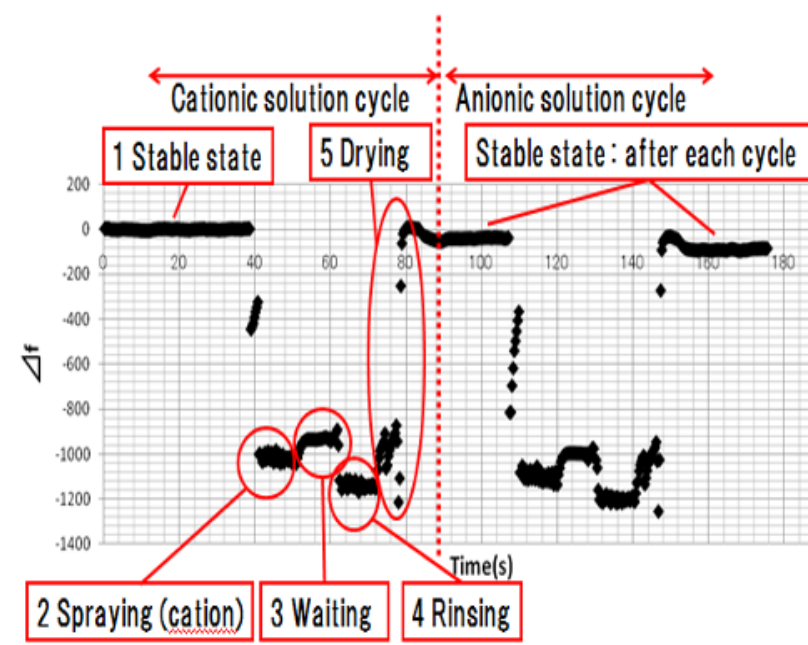

Figure 2. In-situ monitoring by QCM, showing one cycle for a (PAH/PAA) film. Five distinct stages were apparent: 1 stable state; 2 spraying state, 3 waiting state, 4 rinsing state, 5 drying state. Stage times are shown in Table 1.

STEP 1: Stable state: The average frequency during the first stable state was defined as the base frequency for subsequent stages.

STEP 2: Spraying state (solution): When solutions were sprayed, the QCM frequency rapidly decreased and there was significant noise. The frequency decreased because of the adsorption of water and other solutions. Pressure on the QCM surface induced significant noise so the spraying state was unsuitable for measuring the adsorbed mass increase.

STEP 3: Waiting state: The frequency increased a small amount and the noise disappeared. As droplet presence was not constant, the waiting state was also unsuitable for estimating the quantity of adsorbed material.

STEP 4: Rinsing state (Pure water):The frequency during the rinsing state was similar to that at the spraying state, and frequency decreased and fell into disorder. Some surplus aggregation was removed, with only absorbed polymer bonded remaining on the quartz surface.

STEP 5: Drying state(10 seconds):The QCM surface was then dried by blowing with air. The frequency rapidly increased as any remaining water was removed, and frequency recovered to near stable state values.

\subsection{Frequency change and Film thickness}

Ten cycles ware carried out and a ten-bilayer film was fabricated on the QCM, silicon wafer and glass slide. The film on the slide glass was flat and transparent. The film mass on the QCM crystal and the film thickness on the silicon wafer were measured and compared using ellipsometry.

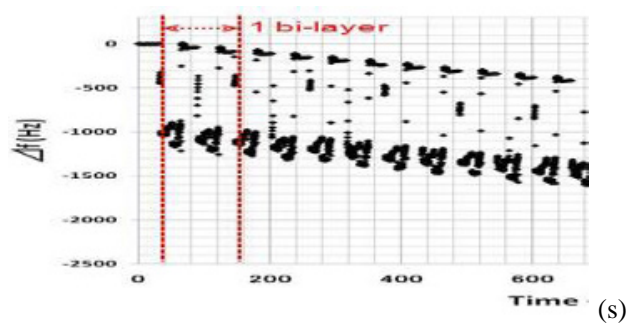

Figure 3. In-situ monitoring by QCM, showing ten cycles of (PAH/PAA) film being prepared. The spraying cycle was repeated using $\mathrm{PAH}$ and $\mathrm{PAA}$, so stable states occurred twenty times.

As shown in Fig.3, the QCM frequency at the stable state linearly decreased, because the frequency change was the same at every bi-layer, and a stable state had occurred at every cycle. It shows the linear growth of film mass with increasing number of bi-layers. At the end of ten cycles, the total frequency decrease was $760 \mathrm{~Hz}$. The silicon wafer film thickness was measured by ellipsometry, and film thickness increased linearly. After ten spray cycles the film thickness was found to be $17 \mathrm{~nm}$. Using the QCM system, film thickness was monitored in real time regardless of substrate type. Quantitative correlation of the frequency change on the QCM and substrate film thickness should be investigated first, and once this relationship is known, reproducible film growth data is always obtained. The high film reproducibility is a significant advantage for industrial applications. The improvement in reproducibility with using the automatic spray-LBL machine is a significant result for optical applications.

\subsection{Enlargement using a movement pattern}

To overcome the ununifirmity problems peculiar to a spray which caused from the various parameters unfixed by analog process, we fabricated the auto-spray-LBL machine controlled by PC which can move the spray nozzle to scan the substrate during flashing out the solutions. We piled the $\left(\mathrm{PDDA} / \mathrm{SiO}_{2}\right)_{10}$ film up on the $5 \mathrm{~cm} \times 7 \mathrm{~cm}$ square slide glass with fixed position or movement pattern to check the nano-order uniformity on the wide area.

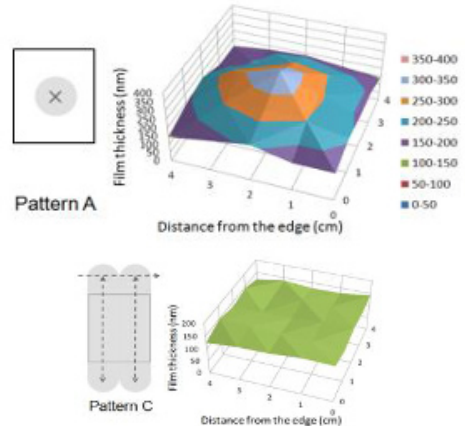

Figure 4. The distribution maps of film thickness 
In the case of pattern $\mathrm{C}$ from Fig 4, which scanned the substrate during the solution spraying with two lines, the uniformity was improved dramatically.

\subsection{High/low refractive index layer}

The $\left(\mathrm{PDDA} / \mathrm{SiO}_{2}\right)_{\mathrm{n}}$ and $(\mathrm{PDDA} / \mathrm{TALH})_{\mathrm{n}}$ film were fabricated. As the increase of number of bi-layer, the film thickness and refractive index were increasing. Llayer/H-layer was piled up to reach the quarter-wave of designed wavelength around 900nm (Table 2).

Table 2 Reflective index and optimal thickness

\begin{tabular}{|c|c|c|c|}
\hline & $\mathrm{n}$ & Reflective index & Thickness \\
\hline $\mathbf{L}:\left(\mathrm{PDDA} / \mathrm{SiO}_{2}\right) \mathrm{n}$ & 14 & 1.33 & $173 \mathrm{~nm}$ \\
\hline $\mathrm{H}:(\mathrm{PDDA} / \mathrm{TALH}) \mathrm{n}$ & 21 & 1.70 & $135 \mathrm{~nm}$ \\
\hline
\end{tabular}

\subsection{Optical characterization and heat shield effect}

Designed H-layer and L-layer were repeatedly stacked on the slide glass without aging. Finally, the film stack was compound $\mathbf{G} / \mathbf{H}(\mathbf{L} / \mathbf{H})_{4}$. Dried at room temperature, fabrication of the Near-IR reflective (NIR-R) film was accomplished. The film thickness was around $1.5 \mu \mathrm{m}$ totally. To investigate the heat shield effect, we fabricate two small boxes enclosed by heat insulator. One side of each boxes, little window adopted to be recessed slide glass was made. Box A; rare slide glass was fit in. Box B; slide glass with NIR-reflective coating was fit in. The two boxes were placed under the nature sun light 10 min after the stabilization.

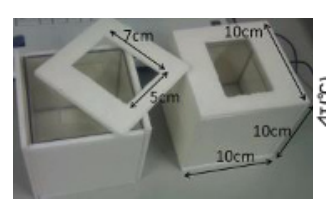

Figure 5. Temperature increase

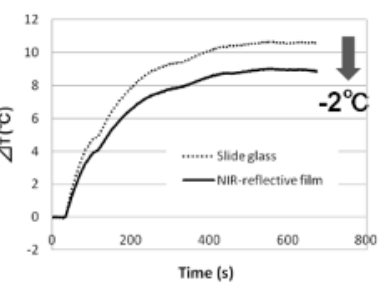

The temperature (T) was measured at a time (Fig.5). The rise of the $\mathbf{T}$ in the Box B which has a NIR-reflective coated window was smaller than the rise of the $\mathbf{T}$ in Box A.

\section{Discussions}

\subsection{Advantages of the Spray deposition}

Compared with the ordinal LBL method by which the substrate is dipped into solution, spray-LBL method was reported that it can fabricate the film quickly. Actually, the spray pressure and the spray time at a certain point of the substrate affect on the film thickness and the refractive index seriously (Fig.1). At a time the solution sprayed on the center of the slide glass, it spreads on the substrate surface radiantly with impinging jet of the air. During spreading of the solution, it seems that the polymers approach to the already piled film surface by spray-LBL method faster than by dipping deposition limited by polymer diffusion. That is why fast the sprayLBL method is.

\subsection{The heat effect by Reflection of Near-IR}

Although heat shield effect was confirmed, the quantity of the effect left the possibility for improvement.
Redesign of optical construction will make the photonic band gap wider, it can improve the heat shield effect.

\section{Conclusion}

We demonstrated enlargement of the coating area by the movement pattern during the spray-LBL deposition with low-cost and eco-friendly process indicated the possibility of the fabrication on the large products such as windowpane. Since the automation and optimization enabled us easily to control the film thickness precisely, we succeeded the production of the technologic optical film such as a Near-IR reflective film which can shield the room from the heat inflow by the sun light. The important advances indicate the development of the spray-LBL method that is sufficient for the industrial application.

\section{References}

1. J. Kim, S. Fujita, S.S. Shiratori, Physicochem. Eng. Aspects 284-385 (2006) 290-294

2. R. Maeda, T. Iwamoto, M. Shakutsui, Journal of Photopolymer Sci. and Tech. 22 (2009) 525-528

3. H. Shimomura,. R. E. Cohen, M. F. Rubner, Appl. Mater. Interfaces 2 (2010) 813-820.

4. J. N. Adam, Michael, M. F. Rubner, R.E. Cohen, Langmuir 20 (2004) 3304-3310.

5. S. K.Srivastava and S. P. Ojha, Microwave and Opt. Tech.38 (2003) 293-297.

6. T. Druffel, N. Mandzy, M. Sunkara, E. Grulke, small 4 (2008) 459-461.

7. D. Yoo, S.S. Shiratori, M. F. Rubner, Macromolecules 31 (1998) 4309-4318: Shiratori, S.S.; Rubner, M.F. Macromolecules 33(2000) 4213-4219.

8. G. Decher, Science 277 (1997) 1232.

9. B. Schlenoff, B. S. Joseph, T. D. Stephan, F, Tarek, Langmuir 16 (2000) 9968-9969.

10. US Patent 6,896,926 B2, (2005); filed 2003.

11. A. Izquierdo, S. S. Ono, J. C. Voegel, G. Decher, Langmuir 21 (2005) 7558-7567.

12. O. Frelix, Z. Zheng, F. Cousin, G. C. R. Decher, Chim. 12 (2009) 225-234.

13. K. C. Krogman, N. S. Zacharia, S. Schroeder, P. T. Hammond, Langmuir 23 (2007) 3137-3141.

14. A. Chunder, K. Etcheverry, S. Wadsworth, D. J. Glenn, Soc. Inf. Display 17 (2009) 389-395.

15. H. Porcel, A. Izquierdo, V. Ball, R. Decher, Langmuir 21 (2005) 800-802.

16. D. Gamboa, A. P. Morgan, A. Ham, Rev. Sci. Instrum. 81 (2010) 036103.

17. K. Ariga, J. Hill, Phys. Chem. Chem. Phys. 7 (2007) 2969-2993.

18. K. H. Kyung, K. Fujimoto, S. Shiratori, J. H. Kim, S. H. Kim, Jap. J. Appl. Phys. 49 (2010) 045001

19. S. S. Shiratori, M. Yamada, Polym. Adv. Technol. 11 (2000) 810-814.

20. N. Fukao, K. H. Kyung, K. Fujimoto. S. Shiratori, Macromolecules 44 (2011) 2964-2969 